\title{
Dentin Phosphoprotein Mimetic Peptide Nanofibers Promote Biomineralization
}

\author{
Gulcihan Gulseren, Gulistan Tansik, Ruslan Garifullin, Ayse B. Tekinay,* \\ and Mustafa O. Guler*
}

Dentin phosphoprotein (DPP) is a major component of the dentin matrix playing crucial role in hydroxyapatite deposition during bone mineralization, making it a prime candidate for the design of novel materials for bone and tooth regeneration. The bioactivity of DPP-derived proteins is controlled by the phosphorylation and dephosphorylation of the serine residues. Here an enzymeresponsive peptide nanofiber system inducing biomineralization is demonstrated. It closely emulates the structural and functional properties of DPP and facilitates apatite-like mineral deposition. The DPP-mimetic peptide molecules self-assemble through dephosphorylation by alkaline phosphatase (ALP), an enzyme participating in tooth and bone matrix mineralization. Nanofiber network formation is also induced through addition of calcium ions. The gelation process following nanofiber formation produces a mineralized extracellular matrix like material, where scaffold properties and phosphate groups promote mineralization. It is demonstrated that the DPP-mimetic peptide nanofiber networks can be used for apatite-like mineral deposition for bone regeneration.

hydroxyapatite within a collagenous matrix. ${ }^{[1-3]}$ Understanding the mechanisms of mineral deposition on collagen is vital for the development of treatments for mineralization-related diseases, and may have key importance for the design of bioinspired materials for hard tissue repair. ${ }^{[1,4-9]}$ Both collagen and noncollagenous proteins modulate the nucleation, growth, and inhibition of hydroxyapatite during hard tissue formation. ${ }^{[2]}$ Among these noncollagenous proteins, dentin sialophosphoprotein (DSPP) is a member of the small integrin-binding ligand, N-linked glycoprotein (SIBLING) family and was originally thought to be dentinspecific. ${ }^{[10]}$ Several studies showed expression of DSPP in bone, ${ }^{[11]}$ cementum, ${ }^{[12]}$ and certain nonmineralized tissues. ${ }^{[13,14]}$ Human and mouse studies demonstrated that DSPP gene mutations and ablations

\section{Introduction}

Mineralization of hard connective tissues such as bone and dentin is a complex process that involves deposition of

Dr. G. Gulseren, Dr. G. Tansik, Dr. R. Garifullin, Prof. A. B. Tekinay

Institute of Materials Science and Nanotechnology

National Nanotechnology Research Center (UNAM)

Bilkent University

Ankara 06800, Turkey

E-mail: atekinay@bilkent.edu.tr

Dr. G. Tansik

Department of Biomedical Engineering

Department of Pathology

DJTMF Biomedical Nanotechnology Institute

University of Miami

Coral Gables, FL 33136, USA

Dr. R. Garifullin

Institute of Fundamental Medicine and Biology

Kazan Federal University

Kazan 420021, Russia

Prof. M. O. Guler

Institute for Molecular Engineering

University of Chicago

Chicago, IL 60637, USA

E-mail: mguler@uchicago.edu

The ORCID identification number(s) for the author(s) of this article can be found under https://doi.org/10.1002/mabi.201800080.

DOI: $10.1002 / \mathrm{mabi} .201800080$ lead to mineralization defects in dentin and bone, which indicates the importance of DSPP in biomineralization. ${ }^{[15-18]}$ As a proprotein, DSPP is activated through cleavage into three distinct proteins; dental phosphoprotein (DPP), dentin sialoprotein (DSP), and dentin glycoprotein (DGP). ${ }^{[19]}$

DPP is abundantly present in the dentin matrix and play crucial roles in the initiation and maturation phases of dentin formation, respectively. ${ }^{[20,21]}$ It has exceptionally high degree of phosphorylation and contains numerous Ser-Asp-Asp, AspSer-Ser, and Asp-Ser repeats, in which the serine residues are potential phosphorylation sites. ${ }^{[22,23]}$ This unique structure allows DPP to facilitate mineral deposition by attracting calcium ions and promoting the formation of hydroxyapatite crystals that form the bulk of the dentin structure. ${ }^{[24]}$ While the hydroxyapatite-binding and mineralization-inducing capacities of DPP have been replicated in a short, serine-rich, nonphosphorylated peptide sequence, ${ }^{[25]}$ the potential effects of serine phosphorylation and dephosphorylation have not been previously studied in biomaterials.

Peptide nanofibers have been widely used since they present various advantages for producing tailored protein inspired materials. ${ }^{[26-29]}$ In this work, we designed a peptide amphiphile molecule (SpDSp-PA) that is capable of emulating the structure and function of DSPP in general and DPP in particular, and studied its capacity to support deposition of hydroxyapatite and mediate the survival and osteogenic differentiation of Saos-2 cells. ${ }^{[30]}$ Like DPP, the SpDSp-PA nanofibers contain 
numerous copies of Ser(phos)-Asp-Ser(phos) sequence in order to effectively induce mineralization and subsequent cellular differentiation. Self-assembled nanofiber formation was induced with two different triggers: through removal of phosphate groups on the SpDSp-PA by ALP; or through neutralization of the peptide with cation addition while a nonphosphorylated control molecule (SDS-PA) was used to study the effect of phosphate groups produced by dephosphorylation process on biomineralization. The SpDSp-PA nanofibers exhibited a strong potential for biomineralization, especially after dephosphorylation by ALP, while soluble peptides were ineffective. In contrast to dephosphorylated SpDSp-PA, nonphosphorylated SDS-PA network failed to facilitate mineral deposition despite their nanofibrous structure, suggesting that phosphate residues and scaffold formation are both critical for the formation of hydroxyapatite like material. ${ }^{[31]}$ The phosphorylated DPP has been demonstrated to display a greater binding affinity to hydroxyapatite due to greater resistance to enzymatic degradation and a greater capacity for calcium deposition. ${ }^{[31-34]}$ Due to their ability to support the survival and osteogenic differentiation of Saos-2 cells, these materials can be used for tissue biomineralization through combination of cellular and mineral components. In addition, we demonstrate the effects of different scaffold formation on biomineralization and osteogenic differentiation. These results are important for designing new complex orthopedic and dental support materials and understanding scaffold effects in regenerative medicine efforts.

\section{Experimental Section}

\subsection{Materials}

4-(2',4'-Dimethoxyphenyl-Fmoc-aminmethyl)-phenoxyacetamido-methylbenzhydryl amine resin (Rink amide MBHA resin), Fmoc-Val-OH, Fmoc-Ala-OH, Fmoc-Gly-OH, FmocSer(PO(OBzl)OH)-OH, Fmoc-Ser(tBu)-OH, Fmoc-Asp(OtBu)$\mathrm{OH}, \quad$ 2-(1H-benzotriazol-1-yl)-1,1,3,3-tetramethyluronium hexafluorophosphate (HBTU), N,N-diisopropylethylamine (DIEA), triisopropylsilane (TIS), trifluoroacetic acid (TFA), $N, N$-dimethylformamide (DMF), dichloromethane (DCM), and diethyl ether were purchased from Merck and Fisher Scientific. Calcein-AM and other cell culture materials were obtained from Invitrogen, Thermo-Fisher and Abcam.

\subsection{Synthesis and Purification of Peptide Amphiphile Molecules}

Lauryl-VVAGS(Phos)DS(Phos)G-Am and Lauryl-VVAGSDSGAm peptides were synthesized on Rink amide MBHA resin at $0.25 \mathrm{mmol}$ scale. Amino acid couplings were done with 2 equivalents of Fmoc-protected amino acid, 1.95 equivalents of HBTU, and 3 equivalents of DIEA for $2 \mathrm{~h}$. Fmoc removal was performed with $20 \%$ piperidine solution in DMF for 20 min. Peptides were cleaved from the resin with a mixture of TFA:TIS: $\mathrm{H}_{2} \mathrm{O}$ in the ratio of $95: 2.5: 2.5$ for $3 \mathrm{~h}$. Excess TFA was removed by rotary evaporation, and the remaining viscous peptide solution was triturated with ice-cold ether. The resulting white product was completely separated from the ether by centrifugation; the centrifugate was dissolved in water, frozen at $-80{ }^{\circ} \mathrm{C}$ and finally freeze-dried. Peptide molecules were characterized by liquid chromatography-mass spectrometry (LC-MS). An Agilent Technologies 6530 Accurate-Mass Q-TOF LC-MS equipped with a Zorbax SB-C18 column was used for LC-MS analysis. The concentration of the sample was $0.5 \mathrm{mg} \mathrm{mL} \mathrm{m}^{-1}$ and samples were analyzed in mobile phase which was water $\left(0.1 \% \mathrm{NH}_{4} \mathrm{OH}\right)$ and acetonitrile $\left(0.1 \% \mathrm{NH}_{4} \mathrm{OH}\right)$.

\subsection{Secondary Structure Analysis of DPP-mimetic PAs}

Secondary structure analysis was carried out following the synthesis and purification of DPP-mimetic peptide amphiphile molecules. All peptides were dissolved in water at a concentration of $1 \times 10^{-3} \mathrm{M}$, and ALP or calcium chloride solution (10-fold excess, $10 \times 10^{-3} \mathrm{M}$ ) were used for gel formation. Peptide/ALP and peptide $/ \mathrm{Ca}^{2+}$ mixtures were incubated until gel equilibrium was reached, and subsequently diluted to a final concentration of $5 \times 10^{-5} \mathrm{M}$ for $\mathrm{CD}$ analysis. CD spectra were recorded on JASCO J-815 spectrophotometer with 3 accumulations and scan speed $100 \mathrm{~nm} \mathrm{~min}^{-1}$.

\subsection{TEM Imaging of DPP-Mimetic PAs}

$1 \times 10^{-3} \mathrm{M}$ peptide solution was prepared and gelled with ALP or $10 \times 10^{-3} \mathrm{M} \mathrm{CaCl}_{2}$ solution and incubated for $6 \mathrm{~h}$. Peptide gels were then diluted with water and a small amount of solutions was dropped to carbon coated copper grids. Peptide fibers were stained with $2 \% \mathrm{w} / \mathrm{v}$ uranyl acetate solution for contrast enhancement: the carbon grids were air-dried prior to TEM measurements. A FEI Tecnai G2 F30 TEM was used for the imaging of peptide amphiphile nanofibers.

\subsection{Enzyme Induced Gel Formation}

$300 \mu \mathrm{L}\left(2 \times 10^{-3} \mathrm{M}\right)$ SpDSp-PA and SDS-PA solutions were prepared, and $1 \mu \mathrm{L}$ ALP $\left(30 \mathrm{U} \mathrm{mg}^{-1}\right.$ ) was added. Enzyme containing peptide solutions and only peptide solutions were incubated in humidified incubators with constant $5 \% \mathrm{CO}_{2}$ at $37{ }^{\circ} \mathrm{C}$ for $2 \mathrm{~h}$. Resulting solution was analyzed with Q-TOF LC-MS system equipped with a reverse phase analytical column. The concentration of the sample was $0.5 \mathrm{mg} \mathrm{mL} \mathrm{m}^{-1}$. The mobile phase was a gradient of water $\left(0.1 \% \mathrm{NH}_{4} \mathrm{OH}\right)$ and acetonitrile $\left(0.1 \% \mathrm{NH}_{4} \mathrm{OH}\right)$.

\subsection{Oscillatory Rheology}

Oscillatory rheology measurements were performed to determine viscoeslastic properties of $\mathrm{Ca}^{2+}$-triggered DPP-like peptide gels with Anton Paar Physica RM301. $25 \mathrm{~mm}$ parallel plate configuration was used for rheometer operating at $25^{\circ} \mathrm{C} .125 \mu \mathrm{L}$ of each sample (peptide) mixed with $125 \mu \mathrm{L} 10$-fold excess calcium chloride solution with a final peptide concentration of $1 \mathrm{wt} \%$ was carefully loaded on the center of the lower plate and 
incubated for $15 \mathrm{~min}$ before measuring. After equilibration, the upper plate was lowered to a gap distance of $0.5 \mathrm{~mm}$. Storage modulus $\left(G^{\prime}\right)$ and loss modulus $\left(G^{\prime \prime}\right)$ values were swept with $10 \mathrm{rad} \mathrm{s}^{-1}$ angular frequency and $0.1 \%$ shear strain. The linear viscoelastic region data for each experimental group were compared after $1 \mathrm{~h}$ time sweep measurement.

\subsection{Surface Mineralization Assay and Energy-Dispersive X-Ray (EDX) Analysis}

Coverslip surfaces were coated with $2 \times 10^{-3}$ M peptide solutions and supplied with $1.5 \times$ simulated body fluid (SBF). After incubation for $12 \mathrm{~h}$, the solution was discarded and the coverslips were washed repeatedly with water, coated with $3 \mathrm{~nm}$ $\mathrm{Au}-\mathrm{Pd}$ and imaged under scanning electron microscope operated at $10-15 \mathrm{keV}$.

EDX spectra analysis was performed to prove presence of calcium $(\mathrm{Ca})$ and phosphate $(\mathrm{P})$ minerals on pPA peptide nanofiber-coated surfaces (Figure S8, Supporting Information). Peaks were collected from $n=3$ coatings per sample and peak intensities collected from each replica were compared to obtain quantitative comparison of mineral deposition among experimental groups.

\subsection{X-Ray Diffractometry Analysis}

The crystallographic structure of calcium phosphate crystals was evaluated with a PAN analytical X'Pert X-ray diffractometer using $\mathrm{Cu} \mathrm{K} \alpha$ radiation. Peptide-coated glass surfaces were prepared as described and measured without further modification. The rotation time was $16 \mathrm{~s}$, scan range was from $20^{\circ}$ to $50^{\circ}$, and step size was $0.013^{\circ}$. The planes observed were (002), (210), (211), (202), (310), (113), (222), (312), and (320) for all of the crystals formed on surfaces.

\subsection{Cell Culture and Maintenance}

Saos-2 human osteosarcoma cells (ATCCHTB-85) were used in viability, immunocytochemistry, and gene expression experiments. All cells were cultured in $75 \mathrm{~cm}^{2}$ cell culture flasks using Dulbecco's modified Eagle medium (DMEM) supplemented with $10 \%$ fetal bovine serum (FBS), $1 \%$ penicillin/streptomycin, and $2 \times 10^{-3} \mathrm{M}$ L-glutamine. The cells were kept at $37^{\circ} \mathrm{C}$ in a humidified chamber supplied with $5 \% \mathrm{CO}_{2}$. Cell passage was carried out at cell confluency between $80 \%$ and $90 \%$ using trypsin/ ethylenediaminetetraacetic acid (EDTA) chemistry. The culture medium was changed every 3-4 days. The osteogenic differentiation experiments (immunocytochemistry stainings and quantitative reverse transcription-polymerase chain reaction (qRT-PCR) analysis) were conducted in two groups: osteogenic medium group [osteogenic medium is a combination of the maintenance medium with $10 \times 10^{-3} \mathrm{M} \beta$-glycerophosphate, $50 \mu \mathrm{g} \mathrm{mL}^{-1}$ ascorbic acid, and $10 \times 10^{-9} \mathrm{M}$ dexamethasone] and maintenance medium group [Dulbecco's modified Eagle's medium (DMEM, Gibco), containing $10 \%$ fetal bovine serum, $100 \mu \mathrm{g} \mathrm{mL}{ }^{-1}$ streptomycin and $100 \mathrm{U} \mathrm{mL}^{-1}$ penicillin/streptomycin].

\subsection{Viability of Saos-2 Cells on Peptide Networks}

The viability of Saos-2 cells incubated on peptide nanofibers and uncoated tissue culture plates (TCP) for $24 \mathrm{~h}$ was studied by Live/Dead Assay (Invitrogen). Briefly, cells were seeded on peptide coated nanofibers and uncoated 96 well tissue culture plates at a density of $5 \times 10^{3}$ cells per well for $24 \mathrm{~h}$. After incubation under standard conditions for $24 \mathrm{~h}$, cells were stained with $2 \times 10^{-6} \mathrm{M}$ Calcein-AM and $4 \times 10^{-6} \mathrm{M}$ ethidium homodimer I (EthD-1) in PBS (1×) for $30 \mathrm{~min}$ at room temperature. After incubation, images were taken at three random points per well with a fluorescent microscope at $10 \times$ magnification. Viable cells were imaged under fluorescence microscope. All samples were studied in triplicate. Live and dead cells were counted by using ImageJ and number of live cells was calculated for each sample.

\subsection{Immunocytochemistry}

Before immunocytochemistry staining, differentiated cells were fixed with $4 \%$ formaldehyde for $15 \mathrm{~min}$ and permeabilized with $0.5 \%$ Triton-X for $10 \mathrm{~min}$ at room temperature. $3 \mathrm{wt} \% \mathrm{BSA} / \mathrm{PBS}$ was used for blocking for $1 \mathrm{~h}$. Rabbit-raised, anti-human, DMP-1 polyclonal antibodies and goat-raised, anti-rabbit, IgG H\&L DyLight 488 conjugated secondary antibody (Abcam) (ab103203 and ab96899, respectively) were obtained from Abcam. The cell nuclei were stained with TO-PRO-3 iodide. The samples were visualized with the Zeiss LSM 510 confocal microscope.

\subsection{Gene Expression Analysis}

For gene expression studies, Saos-2 cells were seeded on peptide nanofiber-coated and uncoated wells of 6 well plates at a density of $2.5 \times 10^{5}$ cells $\mathrm{cm}^{-2}$. Gene expression profiles of Runx2, Collagen I, and osteopontin were evaluated by qRT-PCR analysis for investigating the osteogenic differentiation process. RNA isolation from Saos-2 cells seeded on peptide nanofiber-coated and bare surfaces was performed after 7 days of incubation by using TRIzol (Invitrogen) according to the manufacturer's instructions. Yield and purity of extracted RNAs were assessed by Nanodrop 2000 (Thermo Scientific). Samples were diluted to a concentration of $100 \mathrm{ng} \mu \mathrm{L}^{-1}$ prior to their use. Primers for PCR amplification of Runx2, Collagen I, osteopontin and GAPDH are shown in Table S1 (Supporting Information). Melting temperatures $\left(T_{\mathrm{m}}\right)$ for the primers were determined as $58{ }^{\circ} \mathrm{C}$ for Runx2 and GAPDH, $57.3^{\circ} \mathrm{C}$ for osteopontin, and $61.4{ }^{\circ} \mathrm{C}$ for Collagen I. cDNA synthesis from RNA and qRT-PCR were performed using SuperScript III Platinum SYBR Green One-Step qRT-PCR Kit according to the manufacturer's instructions. mRNA levels were calculated and normalized to GAPDH according to comparative Ct method for each target gene. ${ }^{[35]}$

\subsection{Statistical Analysis}

All quantitative values are presented as mean \pm SEM (standard error of mean), and all experiments were performed with at 


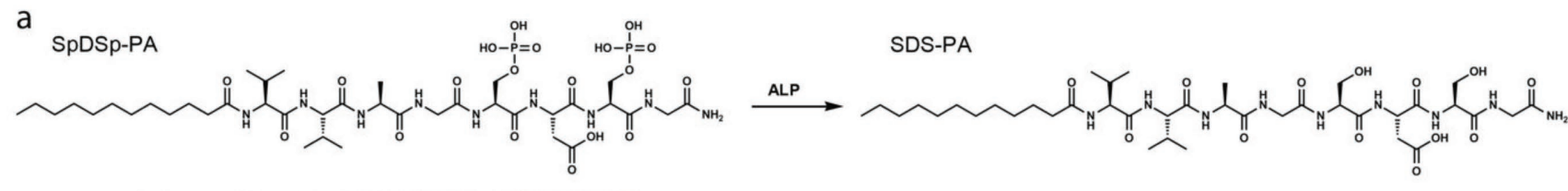

b

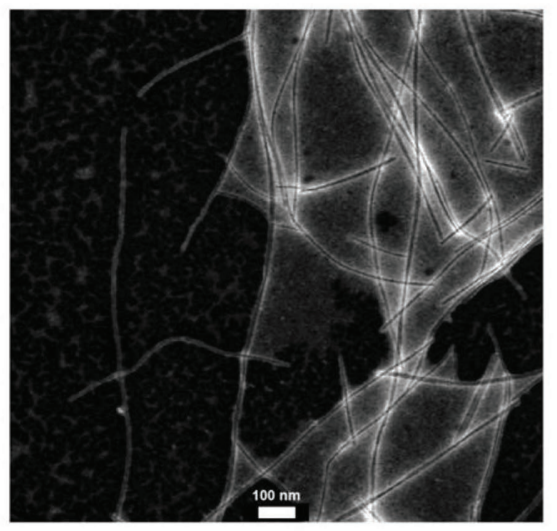

d

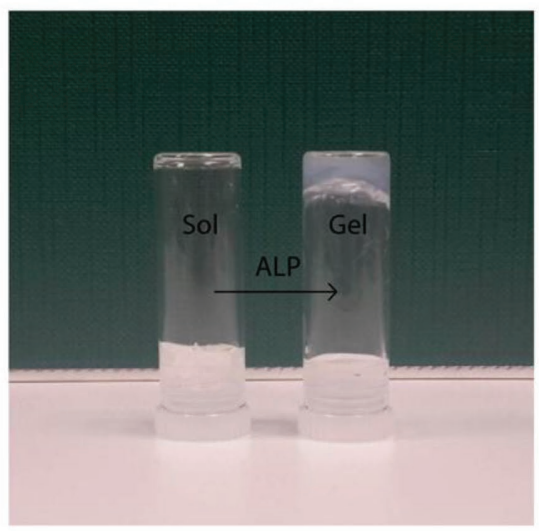

C

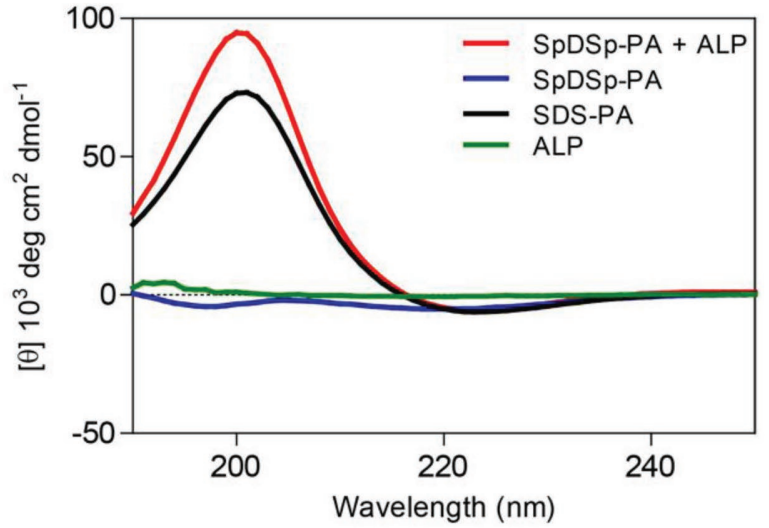

e

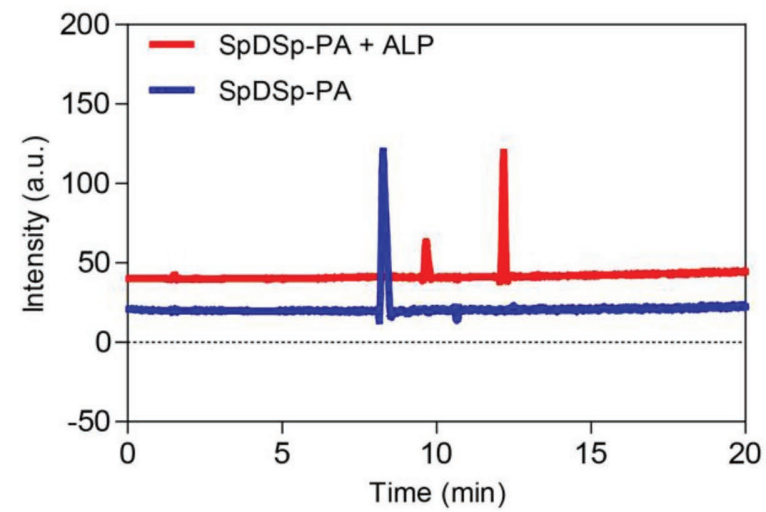

Figure 1. Enzymatic cleavage and gel formation of DPP-like peptides. a) Enzymatic dephosphorylation process of SpDSp-PA with ALP. b) TEM Images of peptide nanofibers formed after ALP treatment. c) Circular dichroism data showing $\beta$-sheet formation before and after enzymatic treatment, and control SDS-PA. d) Illustration of enzyme induced gel formation and e) LC-MS analysis of enzymatic cleavage process.

least three replicates. One-way analysis of variance (ANOVA) was used for the statistical analysis of viability tests, gene expression studies and $\mathrm{Ca}^{2+}$ deposition quantification analyses. All statistical tests were performed using Graph-pad Prism v5.0.

\section{Results and Discussion}

\subsection{Design and Characterization of DPP-Mimetic Peptide Amphiphile Molecules}

Both phosphorylated and nonphosphorylated peptide amphiphiles were designed with the Lauryl-VVAGSDSG-Am sequence to mimic the Ser-Asp-Ser motifs present in large numbers in DSPP and DPP. The hydrophobic lauryl group acts in conjunction with the $\beta$-sheet-forming VVAG motif to enhance the self-assembly of the peptide into nanofibers, while the SDSG sequence presents phosphorylated or nonphosphorylated serine residues to facilitate the deposition of hydroxyapatite on the nanofiber matrix. This sequence was synthesized with and without phosphorylated serine residues through the inclusion of the appropriate amino acid during the synthesis, and molecules were named as SDS-PA (for nonphosphorylated PA) and SpDSp-PA (for phosphorylated PA) (Figure 1a). Following purification and identification of the peptides by LC and MS (Figure S1, Supporting Information), the physicochemical characteristics of SDS-PA, SpDSp-PA, and their gels were characterized by circular dichroism (CD) spectroscopy, oscillatory rheology, scanning electron microscopy (SEM), and transmission electron microscopy (TEM).

Self-assembly of SpDSp-PA molecules was triggered both by ALP-mediated dephosphorylation and calcium ion addition (Figure S2, Supporting Information). Dephosphorylation of SpDSp-PA resulted in cleavage of the negatively charged phosphate groups that normally prevent the self-assembly of the peptide, while calcium ion addition promoted the nanofiber formation through charge screening and electrostatic crosslinking by divalent metal ion. Both ALP and $\mathrm{Ca}^{2+}$ treated solutions were observed to rapidly form gels at room temperature and exhibited CD spectra consistent with a $\beta$-sheet structure (Figures $1 \mathrm{~b}, \mathrm{~d}$ and $3 \mathrm{a}, \mathrm{b}$, and Figure S4, Supporting Information). In contrast, SpDSp-PA did not exhibit any $\beta$-sheet formation in water due 

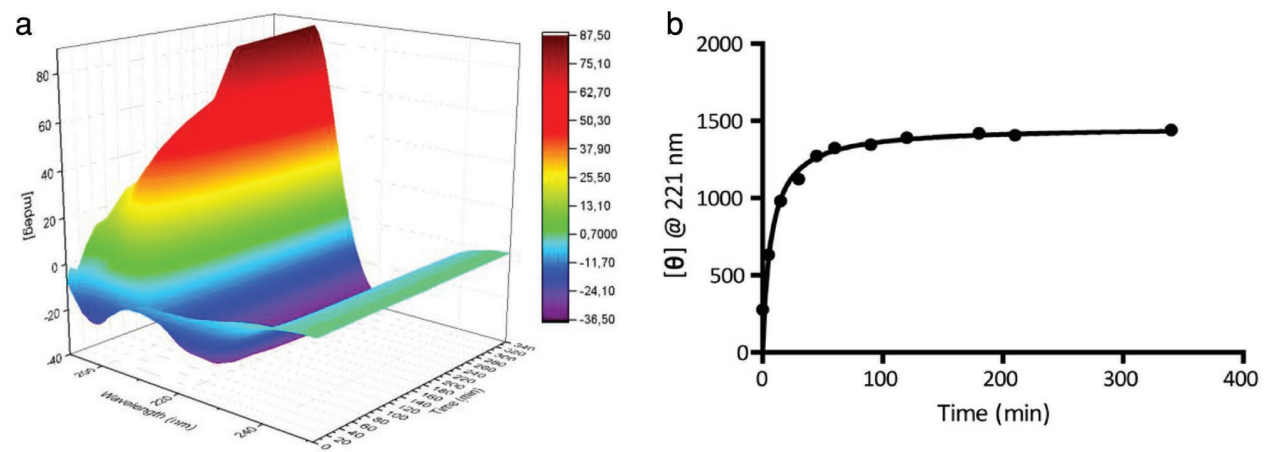

Figure 2. In-depth monitoring of network formation by dephosphorylated SpDSp-PA. The $\beta$-sheet formation rate was monitored by biocatalytic conversion of the SpDSp-PA. a) Time-course circular dichroism measurement of ALP-treated SpDSp-PA showing gradual formation of $\beta$-sheet signal. b) Quantification of the $221 \mathrm{~nm}$ signal of $\beta$-sheet structure showing that dephosphorylation is complete in $1 \mathrm{~h}$.

to its strong negative charge preventing self-assembly, while SDS-PA had a CD signal similar to that of the ALP-treated SpDSp-PA.

The enzymatic dephosphorylation of SpDSp-PA was monitored in real time through LC-MS and CD measurements. Unmodified SpDSp-PA eluted at $8 \mathrm{~min}$ in water/acetonitrile gradient due to its strong negative charge, while the ALPtreated group was observed later (at $\approx 12 \mathrm{~min}$ ) due to removal of the phosphate groups (Figure 1e). Completely dephosphorylated SpDSp-PA had mass spectrum similar to that of synthetic SDS-PA, confirming successful dephosphorylation (Figure S3, Supporting Information). In addition, time-lapse CD measurements demonstrated that a negative signal appears at $221 \mathrm{~nm}$ in the CD spectrum of SpDSp-PA following ALP-treatment, and gradually increases with time (Figure 2a). This signal is associated with the $\beta$-sheet formation and corresponds to the self-assembly of the SpDSp-PA, concurrent with its dephosphorylation by ALP. The $221 \mathrm{~nm}$ signal reached a plateau at $1 \mathrm{~h}$ suggesting that dephosphorylation and self-assembly are complete (Figure 2b). Kinetics of the self-assembly was monitored by nanofiber formation with half-life of $7.37 \mathrm{~min}$. Then, the system slowly reached equilibrium in accordance with decreasing precursor. The rate of catalytic self-assembly could be tuned simply by changing the amount of enzyme present in the system. Concentration-dependent CD measurements also suggested that SpDSp-PA can form $\beta$-sheet structures at concentrations as low as $\approx 5 \times 10^{-6} \mathrm{M}$ (Figure S4, Supporting Information).

Calcium ion-induced gel formation was also studied by TEM, $\mathrm{CD}$, and oscillatory rheology. While SDS-PA did not substantially react to the addition of calcium ion in CD measurements, SpDSp-PA exhibited a substantial increase in the intensity of $\beta$-sheet-associated CD signal at $221 \mathrm{~nm}$, as the negatively charged phosphoserine residues in its structure allowed it to form self-assembled nanostructures following the addition of calcium ions (Figure 3a). In contrast, SDS-PA did not require a neutralizing agent for its self-assembly, and consequently maintained a $\beta$-sheet structure with or without the inclusion of a cation. However, it should be noted that cation inclusion supports self-assembly and nanofiber stability due to the negative charge on the peptides. It is notable that the storage modulus of $\mathrm{Ca}^{2+}$ treated SpDSp-PA was substantially higher than that of $\mathrm{Ca}^{2+}$ treated SDS-PA, suggesting that ion-mediated assemblies of phosphorylated peptides are mechanically more rigid. Phosphate groups on the nanostructure improve cation mediated self-assembly (Figure 3c and Figure S5, Supporting Information). Nevertheless, both hydrogel forms exhibited a nanofibrous structure similar to many peptide amphiphile assemblies (Figures 3b,d).

\subsection{Mineral Deposition Capacity of DSPP-Mimetic PAs under Enzymatic and lonic Self-Assembly}

Following the material characterization of SpDSp-PA, SDS-PA, and their self-assembled forms, an in vitro biomineralization assay was performed to study the formation of a mineralized matrix. Interestingly, the ALP-treated SpDSp-PA group exhibited a very high rate of biomineralization, heavily coating the glass coverslip with calcium depositions matching the $\mathrm{Ca} P \mathrm{P}$ ratio of hydroxyapatite (Figure 4 and Figure S6, Supporting Information). Ca:P ratio of all groups was demonstrated to understand the structure of crystalline content on each experimental group (Figure 4). $\mathrm{Ca}^{2+}$-treated SpDSp-PA also supported biomineralization, although with lower crystalline content (Figure S7, Supporting Information). Nontreated SpDSp-PA and SDS-PA were both largely ineffective in facilitating the mineralization of the glass surface. Effective hydroxyapatite deposition in enzyme treated group is in contrast to prior studies suggesting that dephosphorylated DPP loses a portion of its affinity for hydroxyapatite, resulting in lower rates of biomineralization (or even the inhibition of mineral deposition) compared to its phosphorylated form. ${ }^{[36,37]}$ It should be noted that free SpDSpPA is in soluble form while the ALP-treated peptide self-assembles into a nanofibrous matrix that may assist in the deposition of hydroxyapatite by presenting a well-ordered series of amino acid residues. We therefore suggest that the presence of a wellorganized nanofiber scaffold can support or substitute for the strongly negative charge of naturally occurring DPP, allowing for efficient mineralization even in the absence of bound phosphoryl groups. In addition, the phosphoryl functional groups removed by ALP may be available for the formation of hydroxyapatite or even remain in association with the peptide matrix to better facilitate the mineral deposition process.

Some of these effects may also apply to the in vivo development of dentin and enamel. For example, the DPP and DSP 


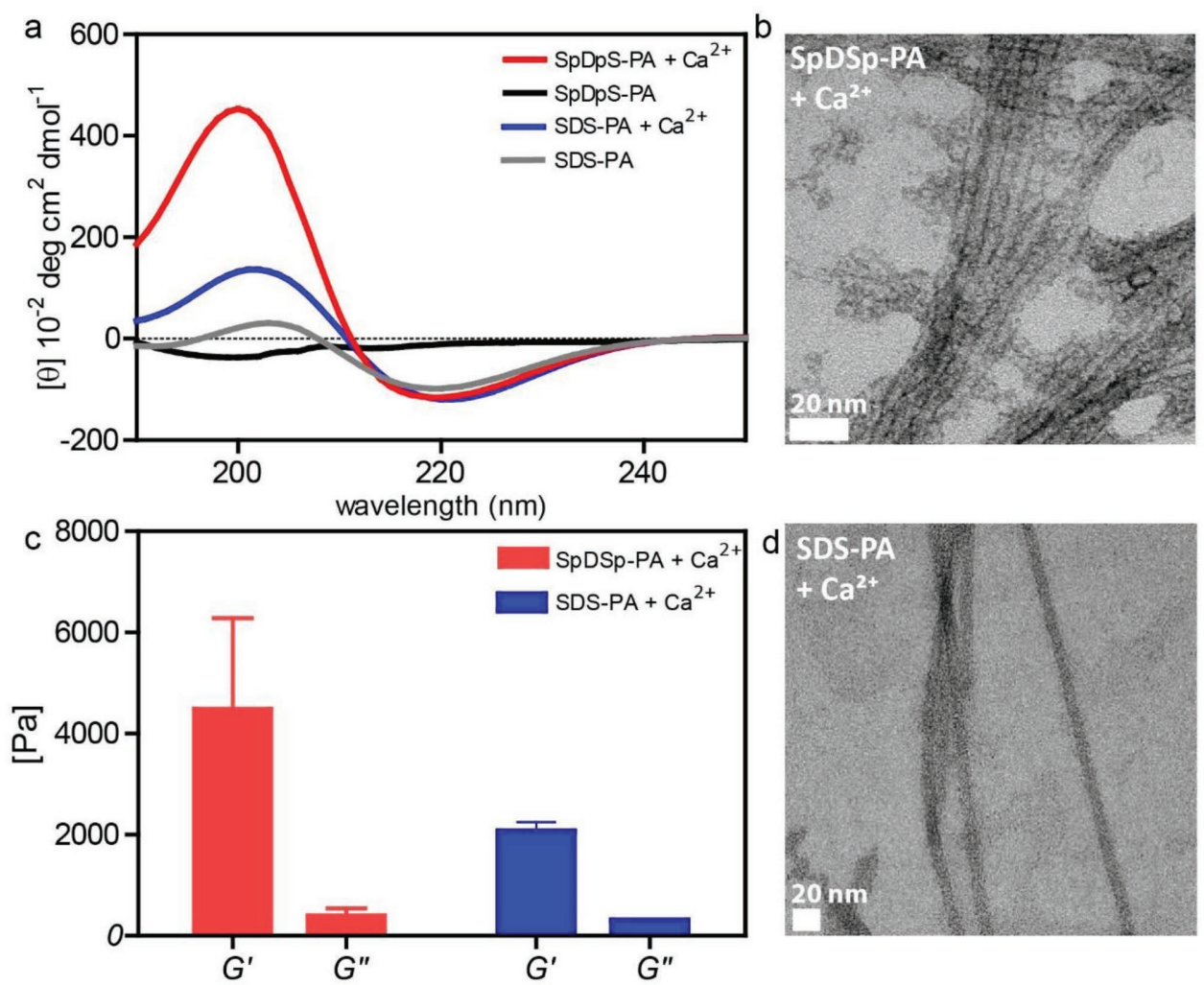

Figure 3. Calcium ion induced gel formation of DPP-like peptides. In-depth monitoring of network formation by calcium-treated SpDSp-PA and SDS-PA. a) Circular dichroism spectra showing $\mathrm{Ca}^{2+}$-triggered self-assembly of SpDSp-PA, and self-assembly of SDS-PA with and without cation presence. b,d) TEM images of SDS-PA $+\mathrm{Ca}^{2+}$ and SpDSp-PA $+\mathrm{Ca}^{2+}$ nanofiber networks. c) Oscillatory rheology of SpDSp-PA $+\mathrm{Ca}^{2+}$ and SDS-PA $+\mathrm{Ca}^{2+}$ nanofiber networks.

in teeth are partially dephosphorylated with age, ${ }^{[38]}$ which results in a lowered capacity for biomineralization that may be mitigated through the presence of a well-established scaffold matrix. In addition, the phosphorylation and dephosphorylation of DPP is constantly regulated by enzymatic activity, and a balance between the two processes is necessary for modulating the mineral content in hard tissues by controlling the concentration of calcium and phosphorus in free and HA-bound forms. ${ }^{[24]}$ As SpDSp-PA can be dephosphorylated, and retains function to act as a bioactive matrix in its dephosphorylated form, we suggest that the present system can serve as a model for further investigation of enzyme activity and its effects on biomineralization. In order to better demonstrate the efficacy of the SpDSp-PA system as a mimic of the native DPP matrix, we further studied osteogenic differentiation of Saos-2 cells on these networks.

\subsection{Cell Viability of Saos-2 Cells on Peptide Nanofibers}

Biocompatibility of the DPP-mimetic PAs was tested with live/ dead assay for Saos-2 cells. Cells were viable on all surfaces over a $24 \mathrm{~h}$ period. No significant difference was observed between the viability of cells on different peptide nanofiber scaffolds and bare tissue culture plate (TCP) suggesting that the peptide nanofibers provided a biocompatible environment for cellular survival (Figures S9 and S10, Supporting Information).

\subsection{Molecular Analysis of Osteogenic Differentiation}

The differentiation of progenitor cells into the osteogenic lineage is regulated by the expression of specific marker genes such as transcription factors, adhesion molecules and proteins of the extracellular matrix. ${ }^{[39]}$ Here, expressions of the osteogenic markers Runt-related transcription factor 2 (Runx2), type I collagen (Collagen I) and osteopontin were studied on day 7 in both osteogenic medium and maintenance medium groups (Figures $\mathbf{5}$ and $\mathbf{6}$ ). Runx2 is often referred to as the key regulator of osteogenic differentiation ${ }^{[40,41]}$ and its expression is typically analyzed during the early phases. In the osteogenic medium group, the expression of Runx2 was upregulated when cells were cultured on ALP-treated SpDSp-PA nanofibers compared to cells on nontreated SpDSp-PA peptide nanofibers, nontreated SDS-PA nanofibers, $\mathrm{Ca}^{2+}$-treated SDS-PA peptide nanofibers, and bare TCP surfaces by $\approx 2.34, \approx 8, \approx 2.3$, and $\approx 8.3$ folds, respectively. The expression of Runx 2 was also enhanced by $\approx 2.37$ folds on ALP-treated SpDSp-PA nanofibers compared to the $\mathrm{Ca}^{2+}$-treated SpDSp-PA group (Figure 5a). Early stage mineral deposition on collagen matrix acts as a template for mineralization and serves as a marker for osteogenic differentiation. ${ }^{[42]}$ The expression of Collagen I was significantly upregulated when Saos-2 cells were cultured on ALP-treated SpDSp-PA nanofibers compared to cells on nontreated SpDSp-PA nanofibers, nontreated SDS-PA nanofibers, $\mathrm{Ca}^{2+}$-treated SDS-PA nanofibers, and bare TCP surfaces by $\approx 7.6, \approx 16, \approx 5$, and $\approx 34$ folds, 

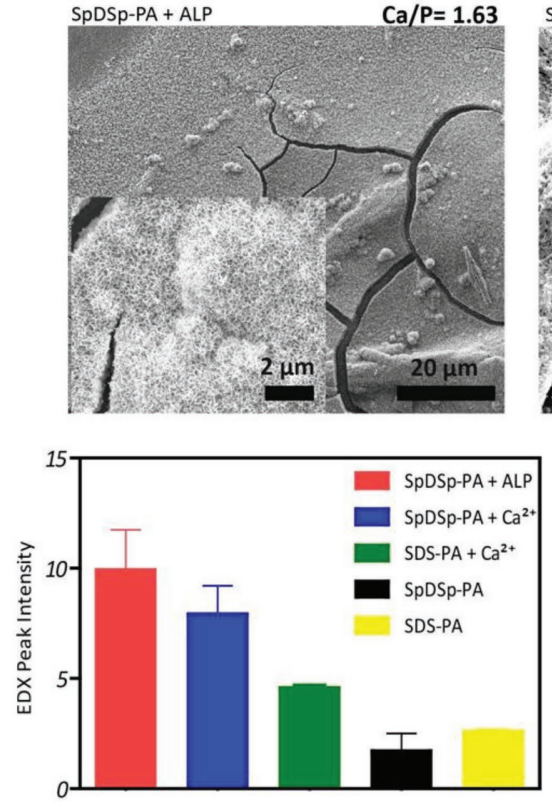
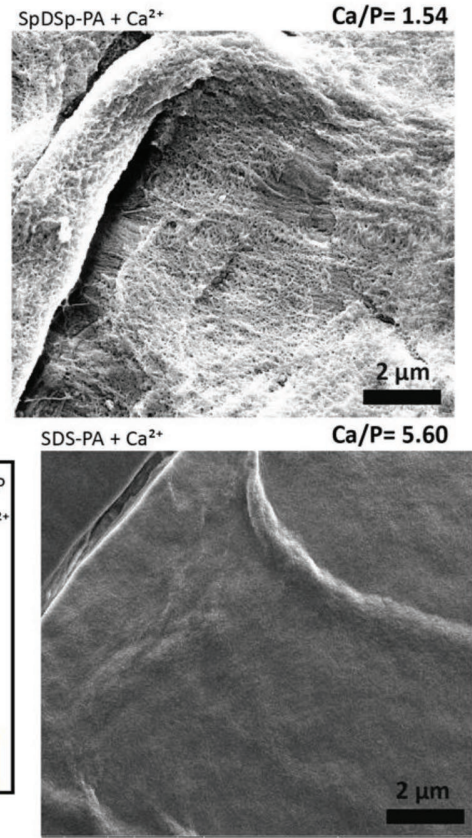

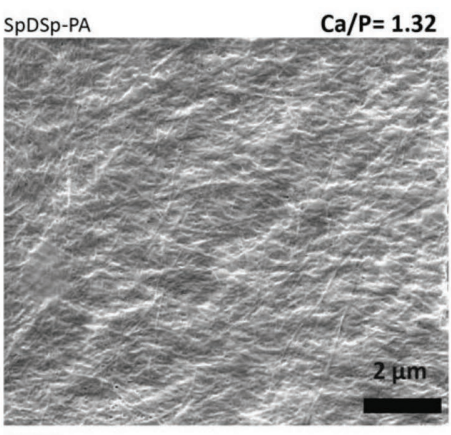

SDS-PA

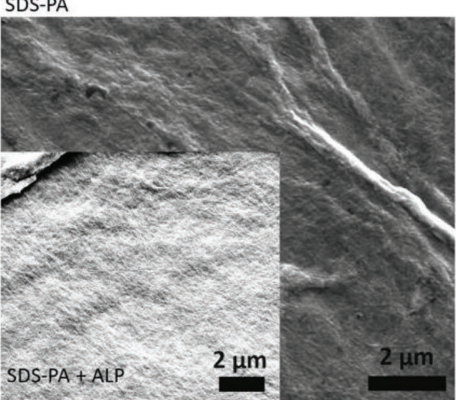

Figure 4. Hydroxyapatite deposition on peptide coated surfaces. Mineral deposition on peptide surfaces with and without nanostructure formation. $\mathrm{Ca} / \mathrm{P}$ ratio was evaluated to reveal mineral composition (above SEM images) of different experimental groups. EDX analysis results of Ca ${ }^{2+}$ content collected from mineralized peptide surface are shown as qualitative mineralization data.

respectively (Figure 5b). The expression of Collagen I was also enhanced by $\approx 3$ folds on ALP-treated SpDSp-PA nanofibers compared to $\mathrm{Ca}^{2+}$-treated SpDSp-PA group.

In addition to Collagen I, several noncollagenous proteins have crucial roles in the formation and maturation of mineralized tissues. Osteopontin is one of the most notable among these proteins and is a highly phosphorylated sialoprotein that occurs as a prominent component of the mineralized extracellular matrices of bones. ${ }^{[43]}$ The osteopontin expression of cells on ALP-treated SpDSp-PA nanofibers was also significantly higher compared to cells on nontreated SpDSp-PA and SDS-PA nanofibers, and bare TCP surfaces by $\approx 3.8, \approx 5.8$, and $\approx 9.6$ folds, respectively (Figure $5 \mathrm{c}$ ). The expression of osteopontin was likewise enhanced by $\approx 1.4$ and $\approx 2$ folds on ALP-treated SpDSp-PA nanofibers compared to $\mathrm{Ca}^{2+}$ treated SpDSp-PA group and $\mathrm{Ca}^{2+}$. treated SDS-PA group, respectively (Figure 5c). Dentin matrix protein 1 (DMP1) is an acidic phosphoprotein that is also present in mineralized tissues and plays an important role in both the intra- and extracellular biomineralization process of osteoblasts and odontoblasts. ${ }^{[44-46]}$ DMP1 expression was also examined on day 14, and immunocytochemistry stainings showed that cells on ALP treated SpDSp-PA nanofibers and $\mathrm{Ca}^{2+}$ treated SpDSp-PA group showed DMP1 staining (Figure 5d). Similar to gene expression analyses, however, the ALP-treated SpDSpPA group was considerably more effective in eliciting DMP1 deposition than the $\mathrm{Ca}^{2+}$ treated SpDSp-PA group.

In contrast to the osteogenic medium results, cells in maintenance medium exhibited enhanced differentiation on $\mathrm{Ca}^{2+}$ treated SpDSp-PA nanofibers compared to ALP treated SpDSpPA as well as other groups tested. In maintenance medium, the expression of Runx2 was significantly upregulated on $\mathrm{Ca}^{2+}$ treated SpDSp-PA nanofibers compared to cells on nontreated
SpDSp-PA peptide nanofibers, nontreated SDS-PA nanofibers, $\mathrm{Ca}^{2+}$ treated SDS-PA nanofibers and bare TCP surfaces by $\approx 9.77, \approx 6.76, \approx 3.6$, and $\approx 22$ folds, respectively. The expression of Runx2 was also significantly enhanced by $\approx 5.17$ on $\mathrm{Ca}^{2+}$ ion-treated SpDSp-PA nanofibers compared to ALP treated SpDSp-PA group (Figure 6a). Similarly, the expression of Collagen I was significantly upregulated when cells were cultured on $\mathrm{Ca}^{2+}$ treated SpDSp-PA nanofibers compared to cells on nontreated SpDSp-PA and SDS-PA nanofibers, $\mathrm{Ca}^{2+}$ treated SDS-PA nanofibers and bare TCP surfaces by $\approx 7.6, \approx 14.6, \approx 2$, and $\approx 25.14$ folds, respectively, and by $\approx 7$ folds compared to the ALP treated SpDSp-PA group (Figure $6 \mathrm{~b}$ ). The expression of osteopontin was also upregulated when cells were cultured on $\mathrm{Ca}^{2+}$ treated SpDSp-PA nanofibers compared to cells on nontreated SpDSp-PA and SDS-PA peptide nanofibers, $\mathrm{Ca}^{2+}$ treated nanofibers and bare TCP surfaces by $\approx 5.6, \approx 2.2, \approx 1.25$, and $\approx 4.29$ folds, respectively, and $\approx 1.91$ folds compared to the ALPtreated SpDSp-PA group (Figure 6c). DMP1 staining results were found to support the gene expression profiles, with cells on $\mathrm{Ca}^{2+}$ treated SpDSp-PA showing enhanced DMP1 expression compared to ALP-treated SpDSp-PA nanofibers, while the TCP group did not show any staining (Figure 6d).

It is interesting to note that Saos-2 cells grown in maintenance medium show enhanced osteogenic gene expression on $\mathrm{Ca}^{2+}$ treated SpDSp-PA compared to ALP treated SpDSpPA, while the reverse trend holds true for cells in osteogenic culture medium. This phenomenon may be attributed to the effect of $\beta$-glycerophosphate, which is a component of the osteogenic medium and promotes osteogenesis by donating free phosphate groups following its cleavage by ALP. As phosphate groups act as both structural components for the biomineralization process and signaling molecules for crucial osteogenic 
a

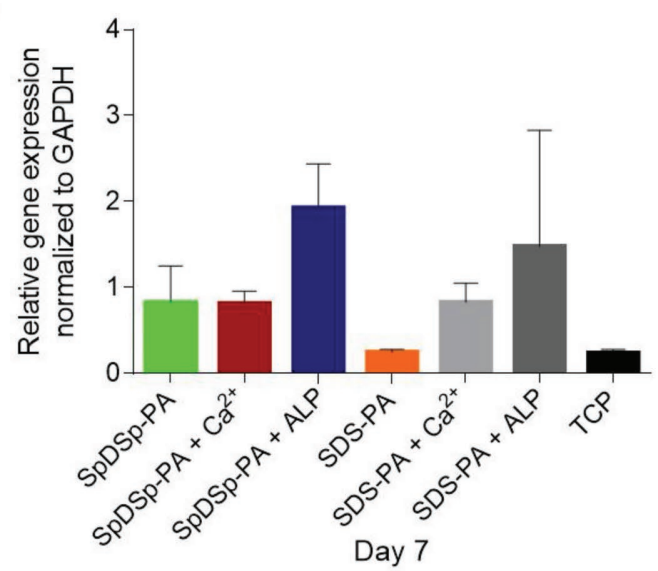

C

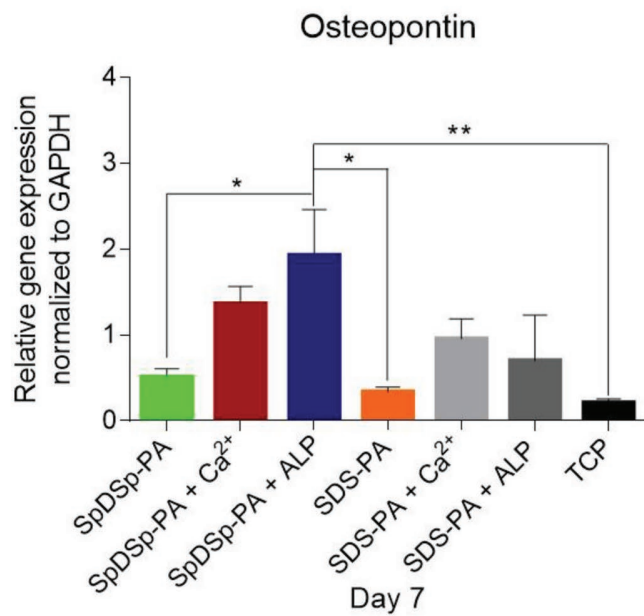

b

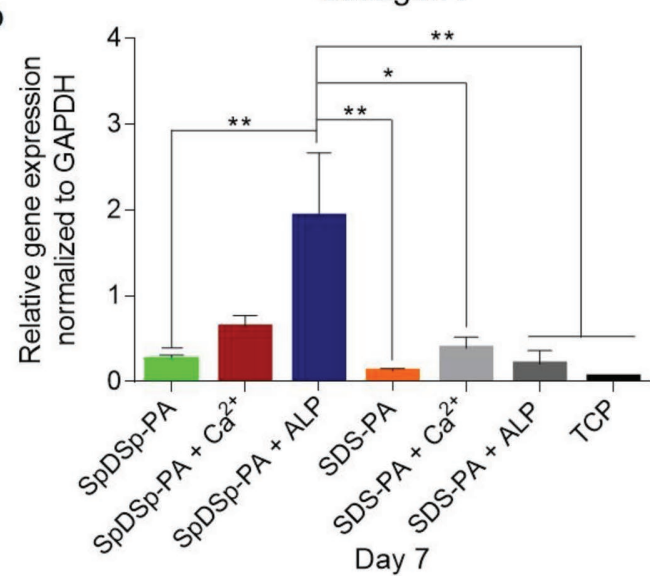

d

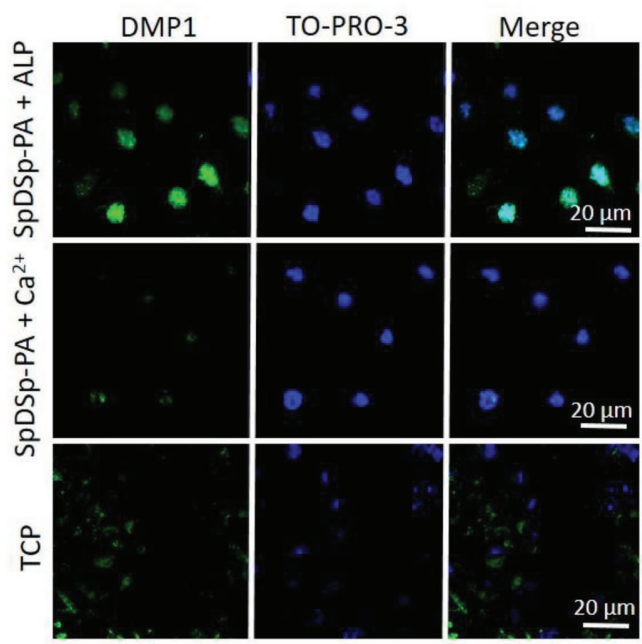

Figure 5. Molecular analysis of osteogenic differentiation in osteogenic medium. Gene expression analysis of Runx2 a), Collagen I b), and osteopontin c) on day 7 of osteogenic differentiation. The expression level of each gene was normalized to GAPDH. Values represent mean \pm SEM ( $* * p<0.01$, $* p<0.05)$. d) Confocal images of DMP-1 immunostaining on day 14. Green shows DMP-1, and blue shows the nucleus.

pathways (such as ERK and cAMP/PKA), the combined effect of phosphate release from $\beta$-glycerophosphate and SpDSp-PA may have provided Saos-2 cells with an enhanced stimulus for osteogenic differentiation, especially in the context of enhanced Runx2 activation by dexamethasone and collagen deposition by ascorbic acid (which are also found in the osteogenic culture medium). Although cells in maintenance medium are also supplied with a free phosphate source on ALP-treated SpDSp-PA, inorganic phosphate by itself is evidently insufficient to promote the matrix modification effects (e.g., collagen deposition, calcification and attachment) that accompany osteogenic development, as evidenced by considerably lower expressions of osteogenic marker genes (and especially Collagen I) on ALP treated SpDSp-PA in maintenance medium. Consequently, cells cultured in maintenance medium are unable to establish their own mineralized matrix on ALP treated SpDSp-PA, and the presence of an existing calcified scaffold in $\mathrm{Ca}^{2+}$ treated SDS-PA is more conducive for osteogenesis through promotion of cell-matrix interactions. Indeed, the presence of a stable scaffold structure is a major contributor to osteogenic differentiation in both osteogenic and normal media, as soluble
SpDSp-PA was relatively ineffective in stimulating osteogenic differentiation under both conditions. Overall, immunostaining and gene expression profiles showed that Saos-2 cells differentiate into osteogenic lineage on both $\mathrm{Ca}^{2+}$ treated and ALP treated SpDSp-PA nanofibers, since enhanced osteogenic differentiation was observed on these peptide nanofiber surfaces. In addition, we observed that different medium conditions may change the differentiation ability of cells, potentially due to the tendency of Saos-2 cells to establish a native matrix in osteogenic medium and rely on their interactions with the existing scaffold in maintenance medium.

\section{Conclusion}

In summary, we showed that DPP-mimetic peptide nanofiber network can mediate biomineralization process through the phosphorylation of its serine residues. DPP-mimetic peptide nanofibers provide a physical scaffold matrix for formation of hyroxyapatite like mineral formation utilizing phosphate and carboxylate groups as a substrate. In contrast, soluble 
a
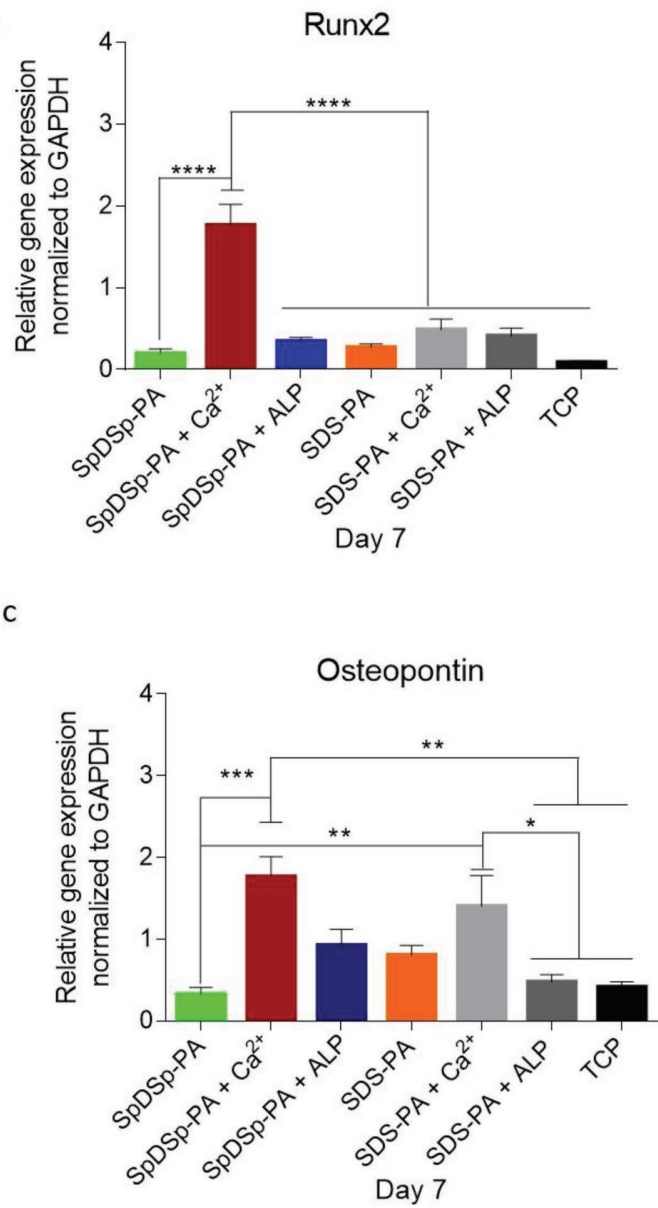

b

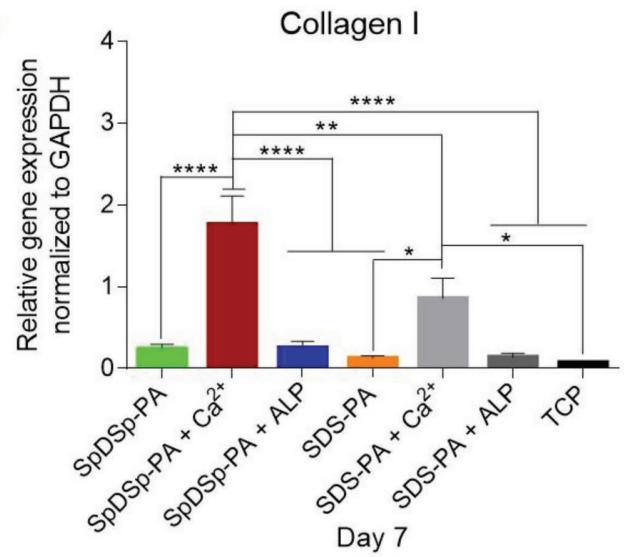

d

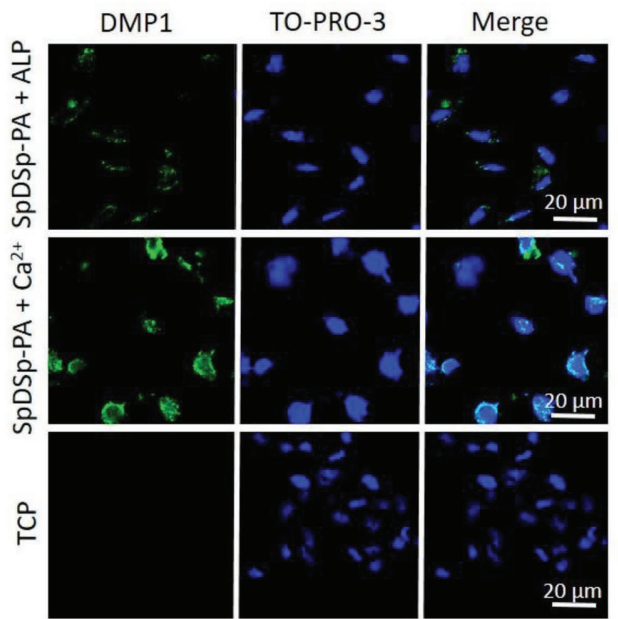

Figure 6. Molecular analysis of osteogenic differentiation in maintenance medium. Gene expression analysis of Runx2 a), Collagen I b), and osteopontin

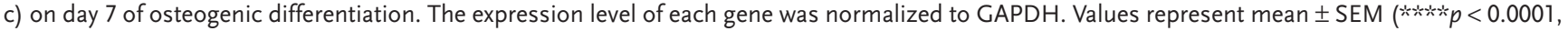
$\left.* k^{*} p<0.001, * * p<0.01, * p<0.05\right)$. d) Confocal images of DMP-1 immunostaining on day 14. Green shows DMP-1, and blue shows the nucleus.

SpDSp-PA and nonphosphorylated SDS-PA nanofibers both failed to facilitate mineral deposition. The presence of phosphate groups and the nanofibrous peptide matrix are both vital for the biomineralization process. In addition, the SpDSpPA and SDS-PA nanofibers supported the viability of Saos-2 cells. The $\mathrm{Ca}^{2+}$ treated SpDSp-PA and ALP treated SpDSp-PA nanofibers enhanced osteogenic differentiation, and they can be utilized to develop new treatments for assisted repair of hard tissue defects because of structural, biochemical and functional resemblance to the DPP.

\section{Supporting Information}

Supporting Information is available from the Wiley Online Library or from the author.

\section{Acknowledgements}

G.G. and G.T. were supported by TUBITAK-BIDEB 2211-C PhD fellowship. R.G. acknowledges RFBR for the grant (16-33-60146) for young researchers and the Program of Competitive Growth of Kazan
Federal University. The authors acknowledge A. D. Ozkan for fruitful discussions and thank Mr. M. Guler for help in TEM imaging.

\section{Conflict of Interest}

The authors declare no conflict of interest.

\section{Keywords}

biomineralization, dentin phosphoprotein, osteogenic differentiation, peptide amphiphile

Received: February 25, 2018

Revised: March 22, 2018

Published online: May 10, 2018

[1] F. Nudelman, A. J. Lausch, N. A. Sommerdijk, E. D. Sone, J. Struct. Biol. 2013, 183, 258.

[2] E. Villarreal-Ramirez, D. Eliezer, R. Garduño-Juarez, A. Gericke, J. M. Perez-Aguilar, A. Boskey, Bone 2017, 95, 65. 
[3] G. Tansik, E. Kilic, M. Beter, B. Demiralp, G. Kiziltas Sendur, N. Can, H. Ozkan, E. Ergul, M. O. Guler, A. B. Tekinay, Biomater. Sci. 2016, 4, 1328.

[4] A. S. Deshpande, E. Beniash, Cryst. Growth Des. 2008, 8, 3084.

[5] Y. Ha, J. Yang, F. Tao, Q. Wu, Y. Song, H. Wang, X. Zhang, P. Yang, Adv. Funct. Mater. 2018, 28, 1704476.

[6] E. D. Eren, G. Tansik, A. B. Tekinay, M. O. Guler, Chem NanoMat 2018, https://doi.org/10.1002/cnma.201700354.

[7] W. Zhang, X. Yu, Y. Li, Z. Su, K. D. Jandt, G. Wei, Prog. Polym. Sci. 2017, 80, 94

[8] G. Wei, Z. Su, N. P. Reynolds, P. Arosio, I. W. Hamley, E. Gazit, R. Mezzenga, Chem. Soc. Rev. 2017, 46, 4661.

[9] J. D. Hartgerink, E. Beniash, S. I. Stupp, Science 2001, 294, 1684.

[10] L. Fisher, D. Torchia, B. Fohr, M. Young, N. Fedarko, Biochem. Biophys. Res. Commun. 2001, 280, 460.

[11] C. Qin, J. Brunn, E. Cadena, A. Ridall, H. Tsujigiwa, H. Nagatsuka, N. Nagai, W. Butler, J. Dent. Res. 2002, 81, 392.

[12] O. Baba, C. Qin, J. C. Brunn, J. E. Jones, J. N. Wygant, B. W. McIntyre, W. T. Butler, Eur. J. Oral Sci. 2004, 112, 163.

[13] K. Alvares, Y. S. Kanwar, A. Veis, Dev. Dyn. 2006, 235, 2980.

[14] K. U. Ogbureke, L. W. Fisher, J. Histochem. Cytochem. 2007, 55, 403.

[15] S. Xiao, C. Yu, X. Chou, W. Yuan, Y. Wang, L. Bu, G. Fu, M. Qian, J. Yang, Y. Shi, Nat. Genet. 2001, 27, 201.

[16] X. Zhang, J. Zhao, C. Li, S. Gao, C. Qiu, P. Liu, G. Wu, B. Qiang, W. H. Lo, Y. Shen, Nat. Genet. 2001, 27, 129.

[17] T. Sreenath, T. Thyagarajan, B. Hall, G. Longenecker, R. D'Souza, S. Hong, J. T. Wright, M. MacDougall, J. Sauk, A. B. Kulkarni, J. Biol. Chem. 2003, 278, 24874.

[18] K. Verdelis, Y. Ling, T. Sreenath, N. Haruyama, M. MacDougall, M. C. van der Meulen, L. Lukashova, L. Spevak, A. B. Kulkarni, A. L. Boskey, Bone 2008, 43, 983.

[19] Y. Yamakoshi, J. Oral Biosci. 2009, 51, 134.

[20] S. Suzuki, T. Sreenath, N. Haruyama, C. Honeycutt, A. Terse, A. Cho, T. Kohler, R. Müller, M. Goldberg, A. B. Kulkarni, Matrix Biol. 2009, 28, 221.

[21] Y. Yamakoshi, J. C.-C. Hu, M. Fukae, H. Zhang, J. P. Simmer, J. Biol. Chem. 2005, 280, 17472.

[22] A. S. Deshpande, P.-A. Fang, X. Zhang, T. Jayaraman, C. Sfeir E. Beniash, Biomacromolecules 2011, 12, 2933.

[23] K. Kawasaki, T. Suzuki, K. M. Weiss, Proc. Natl. Acad. Sci. USA 2004, $101,11356$.
[24] S. L. Lee, A. Veis, T. Glonek, Biochemistry 1977, 16, 2971.

[25] Y. S. Choi, J. Y. Lee, J. S. Suh, G. Lee, C. P. Chung, Y. J. Park, J. Biomed. Mater. Res., Part A 2013, 101, 590.

[26] G. Gulseren, M. A. Khalily, A. B. Tekinay, M. O. Guler, J. Mater. Chem. B 2016, 4, 4605.

[27] G. Gulseren, I. C. Yasa, O. Ustahuseyin, E. D. Tekin, A. B. Tekinay, M. O. Guler, Biomacromolecules 2015, 16, 2198.

[28] E. Arslan, I. C. Garip, G. Gulseren, A. B. Tekinay, M. O. Guler, Adv. Healthcare Mater. 2014, 3, 1357.

[29] A. E. Topal, G. Tansik, A. D. Ozkan, M. O. Guler, A. Dana, A. B. Tekinay, Adv. Mater. Interfaces 2017, 4, 1700090.

[30] Y. Sun, Y. Lu, S. Chen, M. Prasad, X. Wang, Q. Zhu, J. Zhang, H. Ball, J. Feng, W. Butler, J. Dent. Res. 2010, 89, 498.

[31] R. Fujisawa, Y. Kuboki, S. Sasaki, Calcif. Tissue Int. 1986, 39, 248.

[32] A. M. Milan, R. V. Sugars, G. Embery, R. J. Waddington, Eur. J. Oral Sci. 2006, 114, 223.

[33] K. Ibaraki, H. Shimokawa, S. Sasaki, Matrix 1991, 11, 115.

[34] R. Fujisawa, Y. Kuboki, S. Sasaki, Calcif. Tissue Int. 1987, 41, 44.

[35] T. D. Schmittgen, K. J. Livak, Nat. Protoc. 2008, 3, 1101.

[36] M. Wallwork, J. Kirkham, H. Chen, S. Chang, C. Robinson, D. Smith, B. Clarkson, Calcif. Tissue Int. 2002, 71, 249.

[37] C. F. Nawrot, D. J. Campbell, J. K. Schroeder, M. Van Valkenburg, Biochemistry 1976, 15, 3445

[38] P. A. Cloos, A. L. Jensen, Biogerontology 2000, 1, 341.

[39] W. Huang, S. Yang, J. Shao, Y.-P. Li, Front. Biosci.: A J. Virtual Libr. 2007, 12, 3068.

[40] C. Ge, W. P. Cawthorn, Y. Li, G. Zhao, O. A. MacDougald, R. T. Franceschi, J. Cell. Physiol. 2016, 231, 587.

[41] F. Otto, A. P. Thornell, T. Crompton, A. Denzel, K. C. Gilmour I. R. Rosewell, G. W. Stamp, R. S. Beddington, S. Mundlos, B. R. Olsen, Cell 1997, 89, 765.

[42] E. Birmingham, G. Niebur, P. McHugh, G. Shaw, F. Barry, L. McNamara, Eur. Cells Mater. 2012, 23, 13.

[43] J. Sodek, B. Ganss, M. McKee, Crit. Rev. Oral Biol. Med. 2000, 11, 279 .

[44] J. Feng, H. Huang, Y. Lu, L. Ye, Y. Xie, T. Tsutsui, T. Kunieda, T. Castranio, G. Scott, L. Bonewald, J. Dent. Res. 2003, 82, 776.

[45] A. George, R. Silberstein, A. Veis, Connect. Tissue Res. 1995, $33,67$.

[46] R. D'souza, A. Cavender, G. Sunavala, J. Alvarez, T. Ohshima, A. Kulkarni, M. MacDougall, J. Bone Miner. Res. 1997, 12, 2040. 